\title{
Contribución a una historia de la distinción próximo-remoto
}

\section{Contribution to a history of the proximate-ultimate distinction}

\author{
GUSTAVO CAPONI \\ Universidade Federal de Santa Catarina | UFSC
}

\begin{abstract}
RESUMEN La contraposición entre causas próximas que explican cómo los fenómenos biológicos ocurren y causas remotas que explican por qué ocurren, fue enunciada por varios evolucionistas anteriores a Mayr; entre ellos: Edward Poulton. Y, aunque los términos usados por éste no fueron los mismos que Mayr utilizó al trazar la demarcación entre Biología Funcional y Biología Evolutiva, la compresión de la distinción entre dos modos de interrogar lo viviente por él presentada se aproxima mucho al tratamiento que Mayr le dio a la dicotomía en cuestión. Mucho más, sobre todo, que aquella simple distinción entre factores próximos y remotos enunciada por otros naturalistas como Arthur Thomson y John Baker.
\end{abstract}

Palabras clave Ernst Mayr - Edward Poulton - Biología Evolucionaria - causas próximas - causas remotas.

\begin{abstract}
The contrast between proximate causes that explain how biological phenomena happen and ultimate causes that explain why they happen was enunciated by several evolutionists before Mayr, including Edward Poulton. And, although the terms used by the latter were not the same used by Mayr to draw the demarcation between Functional and Evolutionary Biology, his comprehension of the distinction between two ways of interrogating the living being, was very close to the treatment gave by Mayr to this dichotomy. In particular, it was much closer than that simple distinction between proximate and remote factors enunciated by other naturalists like Arthur Thomson and John Baker.
\end{abstract}

Key words Ernst Mayr - Edward Poulton - Evolutionary Biology - proximate causes - ultimate causes.

\section{Presentación}

En la forma y alcance que Ernst Mayr (1904-2005)1 le dio en su paper de 1961, “Cause and effect in Biology”, 2 la distinción entre una Biología Funcional de causas próximas y una Biología Evolucionaria de causas remotas ${ }^{3}$ se transformó en una tesis clásica de la Filosofía de la Biología; ${ }^{4}$ y también se erigió en una referencia epistemológica ineludible para la propia Biología Evolucionaria. ${ }^{5}$ Aunque no hayan faltado, o no falten, los críticos que pretendan, 0 que hayan pretendido, impugnarla, ${ }^{6}$ relativizarla, ${ }^{7}$ flexibilizarla,${ }^{8}$ o reformularla ${ }^{9}$ dicha dicotomía continúa siendo citada cuando se hace necesario circunscribir o establecer la relevancia y la pertinencia evolutiva de una cuestión biológica. ${ }^{10}$ Con todo, mucho antes de adquirir ese estatuto de genuino hito epistemológico, posibilitado justamente por el tratamiento que Mayr le dio, esa polaridad ya había recorrido un camino relativamente largo dentro de la propia Biología Evolucionaria; y buscando sus precedentes, podemos llegar hasta los propios albores de la ciencia moderna. ${ }^{11}$

No será ese último, sin embargo, el objetivo de este trabajo. Sin ir tan lejos, y sin pretender ser exhaustivo en mi estudio, me limitaré a examinar algunos de los precedentes evolucionistas del planteo de Mayr. Aludiré así a John Baker (1900-1984) ${ }^{12}$ y a David Lack (1910-1973) ${ }^{13}$ pero también me referiré a autores que Mayr no menciona entre 
sus precursores. Tal el caso de Edward Albert Sharpey-Schäfer (1850-1935) $)^{14}$ y de Arthur Landsborough Thomson (1890-1977)..$^{15}$

Hasta ahí y salvando un par de análisis más pormenorizados sobre algunos puntos de vistas de esos naturalistas, mi trabajo, lo reconozco, no irá mucho más allá de lo que John Beatty ya señaló, aunque más someramente, en su artículo "The proximate/ultimate distinction in the multiple careers of Ernst Mayr". ${ }^{16}$ Donde sí iré claramente más allá de Beatty es al mostrar de qué manera la contraposición entre causas próximas que explican el cómo del fenómeno biológico, y causas remotas que explican su porqué, ${ }^{17}$ ya había sido claramente formulada, aunque no exactamente en los mismos términos que Mayr, por Edward Poulton (1856-1943):18 ese influyente pionero del Programa Adaptacionista ${ }^{19}$ al cual, por diversas razones, los historiadores de la Biología Evolucionaria aun no le han prestado la atención que ciertamente merece. ${ }^{20}$

Más aun: aunque los términos usados por Poulton no son, conforme digo, los mismos que Mayr utilizó al trazar su demarcación entre Biología Funcional y Biología Evolutiva, la compresión que aquél tenía de la distinción entre dos modos de interrogar lo viviente se aproximaba mucho del tratamiento que medio siglo después le fue dado a esa cuestión en Cause and effect in Biology. Mucho más, sobre todo, de lo que se aproximaba al enfoque de Mayr aquella simple distinción entre factores próximos y remotos enunciada por John Baker: ${ }^{21}$ autor al cual Mayr, ${ }^{22}$ empero, tiende a atribuirle la primera enunciación clara de esa partición cuya historia aquí nos ocupará. ${ }^{23}$

\section{Una primera aproximación a la distinción próximo-remoto}

Sin entrar aun en el análisis histórico, y con el único objetivo de tornar más clara la dicotomía próximo-remoto, me permitiré ensayar una presentación de la misma que no es, siquiera, la del propio Mayr. Entiendo, sin embargo, que esa presentación que yo quiero ensayar, no sólo fortalece teóricamente la polaridad en análisis - cosa que aquí, en realidad, no me incumbe hacer -; sino que además la hace más comprensible. Y eso sí que importa aquí: sobre todo si, al hacerlo, nos precavemos de no distorsionar lo que el propio Mayr, y sus predecesores, querían realmente apuntar al distinguir entre causas próximas y remotas de los fenómenos biológicos. En todo caso, lo que sí haré será aclarar lo que Mayr, Poulton, y otros autores, dijeron a ese respecto, circunscribiéndolo dentro de un esquema conceptual más general que, según entiendo, facilita la comprensión de lo que ellos querían indicar. Las elucidaciones conceptuales, propias de la reflexión epistemológica, siempre pueden auxiliar, de ésta y de otras maneras, al análisis histórico.

Pienso, en este sentido, que la contraposición entre una Biología Funcional de causas próximas y una Biología Evolucionaria de causas remotas es mejor entendida en virtud de la distinción entre organismos y linajes. ${ }^{24}$ La Biología Funcional puede ser descripta como abarcando todo el amplio conjunto de disciplinas biológicas ocupadas en el estudio de las causas próximas que, actuando a nivel de los organismos individuales, nos explican, no sólo cómo los fenómenos vitales se encadenan e integran en la constitución [desarrollo] y el funcionamiento [fisiología] de esas estructuras; sino que también nos permiten entender cómo esos organismos individuales interactúan con su medio biótico y abiótico. La Biología Evolucionaria, mientras tanto, sería esa otra Biología ocupada en indagar las causas remotas que, actuando sobre los diferentes linajes de seres vivos, nos explicarían por qué éstos presentan los caracteres que efectivamente ahora presentan 0 alguna vez presentaron. ${ }^{25}$

Las causas remotas, podemos entonces decir, modifican estados de organismos; las causas remotas, mientras tanto, modifican estados en los caracteres de linajes. Y es en dichos estados que los efectos de las causas remotas se verifican. Lo que produce un cambio en un organismo, lo nutre, lo daña o lo mata, no es una causa remota: es una causa próxima. Aun cuando, sin esas causas próximas que afectan a los organismos individuales, tampoco existirían las causas remotas que afectan a los linajes. Estoy suponiendo, en suma, que la Biología Funcional incluye, no sólo a la Fisiología y a la Biología del Desarrollo; ${ }^{26}$ sino que ella también incorpora a la Autecología y a la Ecología de Poblaciones tout court. ${ }^{27}$ Hecho este último que no siempre queda claro. ${ }^{28}$ 
Mayr y sus predecesores tendieron a superponer el análisis ecológico relativo a las ventajas adaptativas conferidas por un rasgo, con la reconstrucción evolutiva de la historia de dicho rasgo ${ }^{29}$ Como si ese análisis ya conllevase esa reconstrucción. ${ }^{30}$ Pero, además de introducir una precisión en lo que atañe a ese punto, también estoy suponiendo que el concepto de causa remota abarca todos los factores de cambio evolutivo previstos por la versión neosintética de la Teoría de la Selección Natural. Aludo a la deriva genética, a la mutación, a la migración, a la selección sexual y a la propia selección natural. ${ }^{31}$ Las presiones selectivas serían, entonces, sólo un tipo de causa remota; y reconocer esto implica ir más allá de lo dicho al respecto por Mayr y sus predecesores. Casi todos ellos identificaron el concepto de causa remota con el concepto de presión selectiva; ${ }^{32}$ y eso hay que tenerlo muy en cuenta cuando se analizan los textos en los que la polaridad próximo-remoto fue presentada.

\section{La identificación entre causas remotas y presiones selectivas}

Entender la polaridad próximo-remoto a partir de la contraposición entre fenómenos organísmicos y fenómenos que ocurren en el plano de los linajes, es algo que surge, sin necesidad de mayores malabares hermenéuticos, de los propios textos de Mayr. ${ }^{33}$ Se puede decir que esa idea está ahí, latente, cuando él dice que, mientras la Biología Funcional estudia las causas próximas que explican la manifestación de un programa genético, la Biología Evolucionaria hace lo propio con la configuración de ese programa. ${ }^{34}$ Siguiendo métodos experimentales más semejantes a los de la Química y la Física, la Fisiología y la Biología del Desarrollo, nos dice Mayr, explican cómo el organismo opera en virtud del programa que pauta su conformación y su funcionamiento. ${ }^{35}$ Por su parte, y siguiendo métodos predominantemente observacionales y comparativos, la Biología Evolucionaria, nos dice también él, narra la historia evolutiva de esos programas que pautan el funcionamiento y la constitución de los organismos individuales. ${ }^{36}$

Pero esa historia evolutiva, conforme la interpretación neosintética de la Teoría de la Selección Natural ya lo prevé, no está exclusivamente pautada por presiones selectivas. ${ }^{37}$ Ella también obedece, en mayor o menor grado, a otros factores tales como los procesos de migración y a la deriva génica, que también deben ser descriptos como responsables de cambios en linajes. Por eso debemos contar a dichos factores como siendo, ellos mismos y con todo derecho, causas remotas. ${ }^{38}$ Causas cuya propia existencia ya supone una población en el sentido evolutivo de la palabra: una población entendida, no como mero conjunto de organismos integrados a una comunidad ecológica, y sí como un linaje: como una secuencia de generaciones. ${ }^{39}$

El lenguaje de Mayr, es verdad, puede parecernos un poco obsoleto: datado y lastrado, sobre todo, por la hoy ya muy cuestionada metáfora del programa genético. ${ }^{40}$ Pero esa forma de hablar, tan típica del darwinismo neo-sintético posterior a 1960, ${ }^{41}$ no tiene porque llevarnos a pensar que Mayr niegue o menoscabe la importancia de esos otros factores evolutivos cuyo reconocimiento nos fue impuesto, conforme acabo de decir, por la propia Nueva Síntesis. ${ }^{42}$ Mayr, de hecho adalid y portavoz en jefe de dicha síntesis, ${ }^{43}$ nunca negó la eficacia de esos factores y siempre los tuvo en cuenta como agentes de los procesos evolutivos. ${ }^{44}$ Sin embargo, y eso no lo quiero negar, a la hora de explicar qué era una causa remota, los terminó excluyendo de la intensión, por él reconocida, de ese concepto; aun estableciendo, incluso, una equivalencia entre los términos 'causa remota', 'causa evolutiva' y 'causa histórica'. 45

Es decir: Mayr termina caracterizando las causas remotas de una forma que no se compadece con el pluralismo causal de la propia Teoría de la Selección Natural que él mismo defiende.$^{46}$ La idea de que, mientras las causas próximas explican el cómo (how) de los fenómenos biológicos, las causas remotas hacen lo propio con el porqué (why) de esos fenómenos ${ }^{47}$ sólo tiene sentido si se homologa 'causa remota' a 'selección natural' 0 a 'presión selectiva', dejando de lado factores evolutivos como la deriva génica y las migraciones. Caso contrario no habría razón para afirmar que una causa remota nos da el porqué de un fenómeno biológico; y menos aún su para qué ${ }^{48}$ La deriva genética puede citarse para explicar una determinada proporción entre alelos, y, en ese sentido, también puede invocarse para explicar un estado de carácter; pero no diríamos que ella nos brinda el porqué de esa característica. 
Mayr, por otra parte, no está solo en esa identificación entre causas remotas y presiones selectivas. David Lack, uno de los fundadores de la Ecología Evolucionaria, ${ }^{49}$ ya había recuperado esa distinción de Baker en su obra The natural regulation of animals numbers, ${ }^{50}$ de 1954; y su modo de explicarla es, en ese punto, totalmente convergente con la explicación que Mayr daría de ella siete años después. ${ }^{51}$ En lo que atañe a las temporadas de cría, el incremento de la cantidad de horas diarias de luz que ocurre en la primavera sería una causa próxima: dicho incremento estimula el crecimiento y la actividad de los órganos sexuales; y eso conduce a los apareamientos. ${ }^{52}$ Pero la mayor disponibilidad de alimentos que ocurre en el verano, y permite el sustento de las crías resultantes de los apareamientos, sería la causa remota que habría fijado esa sintonización entre luminosidad y actividad sexual.

"Los factores últimos", dice Lack, "tienen que ver con el valor de sobrevivencia, los factores próximos con adaptaciones fisiológicas y comportamentales"; 53 y esa misma idea quedará más clara aun en su discurso presidencial ante la British Ecological Society, proferido el 5 de Enero de 1965. Allí, e invocando otra vez a Baker, Lack dirá que “las características adaptativas deben ser interpretadas en dos niveles, proximalmente en términos de los factores fisiológicos y comportamentales que las generan en el ave individual, y remotamente [ultimately] en términos de su valor de sobrevivencia para la especie". ${ }^{54}$ Lack también entendía, además, que el conocimiento de esas causas remotas permitía responder preguntas por qué y para qué..$^{55}$

Hay que decir, sin embargo, que Baker no apuntó la distinción próximo-remoto con la misma intención de Mayr y de Lack. Éstos lo hacen para reivindicar los derechos de las preguntas y explicaciones propias de una perspectiva evolucionista. Mayr lo hace en general; y lo hace, sobre todo, para reivindicar los derechos de la Biología Evolucionaria frente a la naciente pero ya poderosa Biología Molecular. ${ }^{56}$ Lack, mientras tanto, lo hace pensando en el caso específico de una naciente Ecología Evolucionaria. ${ }^{57}$ Sin que esto último implique negar que el texto de Mayr también acabase siendo muy importante en la autocomprensión epistemológica de esa disciplina. ${ }^{58}$ Baker, en cambio, aun sin querer negar el interés y la legitimidad de dichas cuestiones y explicaciones, tampoco las aborda; limitándose a señalarlas. ${ }^{59}$

Lo que Baker en realidad quiere es mostrar que el trabajo de campo resulta imprescindible para comprender tanto las causas remotas como las causas próximas que regulan las temporadas de cría. Baker quiere mostrar, en otras palabras, que el puro trabajo experimental, hecho por el fisiólogo de laboratorio, es insuficiente para entender dichos procesos. ${ }^{60}$ Más que como un abogado de la Biología Evolucionaria, Baker habla, antes que nada, como un naturalista de campo que quiere reivindicar sus botas embarradas en contra de la pedantería limitante de los biólogos de delantal. ${ }^{61}$ Es claro, sin embargo, que al hablar de factores remotos, Baker tampoco piensa en otra cosa que en presiones selectivas. ${ }^{62}$

Donde sí, en cambio, hay una diferencia a ese respecto es en Problems of bird-migration de Arthur Landsborough Thomson. ${ }^{63}$ También interesado en la migración y en las temporadas de cría de las aves, este naturalista tampoco dejó de apuntar la dicotomía próximo-remoto como siendo una coordenada clave para ese estudio. ${ }^{64}$ Pero lo hizo en términos un poco diferentes a los que después encontramos en Mayr, en Lack y en Baker. Lo que el análisis de Thomson tiene de particular, si comparado con los análisis de esos otros naturalistas, es que para él existe un divorcio claro entre explicación evolutiva y explicación ecológica. Para él, incluso, la causa remota sería algo diferente de lo que hoy llamaríamos una presión selectiva. ${ }^{65}$

\section{Las causas remotas pensadas en clave lamarckiana}

Más allá de las múltiples cuestiones empíricas ahí abordadas, uno de los tópicos centrales de Problems of birdmigration, es la correcta delimitación de esas cuestiones que Thomson considera como los cuatro problemas fundamentales que deben ser resueltos, y nunca confundidos, en el estudio de las migraciones estacionales de las aves. ${ }^{66}$ El primero sería identificar la ventaja ecológica que ese comportamiento conllevaría; y el segundo, reconstruir su historia evolutiva. El tercero, mientras tanto, tendría que ver con la identificación del estimulo que desencadena ese comportamiento y el cuarto con los factores que lo guían durante su ocurrencia. Para Thomson, en suma, los problemas a ser 
considerados en el estudio de las migraciones estacionales de las aves envuelven cuatro tipos de factores posibles que deben ser cuidadosamente distinguidos:

[A] factores que, sin ser realmente causadores, pueden hacer que la migración sea ventajosa, brindándole un valor de sobrevivencia; [B] factores que en el pasado pueden haber ayudado a originar y a desarrollar esa costumbre en la raza; [C] factores que periódicamente estimulan que dicha costumbre se exprese en el individuo en la estación adecuada [...]; y [D] factores que determinan la manera en la que la migración es efectivamente ejecutada. ${ }^{67}$

En lo que atañe al primer problema, Thomson apunta que, siendo la migración una 'costumbre [custom] racial' costosa que conlleva un gran dispendio de energía y redunda en muchas pérdidas de vida, es de esperarse que ella tenga alguna utilidad [useful end] que compense esas desventajas. ${ }^{68}$ "Caso contrario" - dice incluso él - "la migración habría ciertamente dejado de existir: los individuos y las especies estacionarias habrían sobrevivido y los migrantes habrían perecido. La costumbre debe tener una raison d'être; y ése, además de ser el primer problema a ser planteado, es probablemente el único para el cual puede ser ofrecida una solución clara". 69

Para Thomson, en efecto, la razón de ser de los comportamientos migratorios no parece muy difícil de identificar: “De un modo general, los fines a los que sirve la migración son obvios. La misma permite que los migrantes habiten dos áreas diferentes en las estaciones en las que cada una de ellas es más favorable".$^{70}$ Las aves migrantes evitan inviernos rigurosos, en los que faltan los alimentos, ${ }^{71}$ y ellas aprovechan los veranos propicios, en los que dichos alimentos abundan..$^{72}$ Thomson, sin embargo, se esfuerza en dejar en claro que, a su entender, la razón de ser, la utilidad del comportamiento migratorio, no explica su ocurrencia. ${ }^{73}$

"El hecho de que exista una buena razón para la migración" - nos dice él - "no explica su ocurrencia, aunque la existencia de fines útiles pueda darle un valor de sobrevivencia a esa costumbre". ${ }^{74}$ No es por el hecho de ser útil que una cosa habrá de ocurrir:

[...] alguna causa debe operar de forma tal que ese fin sea alcanzado"; y "en el caso de una costumbre congénita, que entra periódicamente en operación, la causación asume un aspecto dual: ¿qué originó y desarrolló la costumbre en la raza, y qué evoca su manifestación periódica en el individuo? ${ }^{75}$

He ahí la distinción entre causas remotas y próximas trazada con toda claridad; y he ahí los problemas segundo y tercero que plantea el estudio de la migración de aves:

[...] el segundo problema alude a la naturaleza de la causa originadora que estableció el comportamiento migratorio en la raza, y el tercer problema alude al estimulo inmediato actuando sobre el individuo que pone esa costumbre en acción cada otoño y cada primavera. ${ }^{76}$

"La solución del primero" - señala incluso Thomson - "debe ser buscada en la historia pasada de la especie de aves en cuestión"; y hasta ahí, claro, sus puntos de vista coinciden con los de Mayr, Lack y Baker. Pero, diferentemente de lo que ocurre con estos últimos, Thomson pensaba esa historia evolutiva de una forma definitivamente neolamarckiana. Para él, el comportamiento migratorio exhibido por un ave individual debía ser entendido como el decantado hereditario de comportamientos ejecutados por sus ancestros a lo largo de generaciones:

Todas las teorías evolucionistas sobre el origen de la migración necesariamente asumen que la ejecución actual de la migración por parte de aves individuales, es el resultado, y en mayor o menor medida la repetición habitual, de un evento o series de eventos ocurridos en su historia racial. Así como el organismo individual en el curso de su desarrollo estructural frecuentemente recapitula etapas de de la evolución del tipo, así las migraciones hoy realizadas por el ave individual pueden reproducir aspectos de algún gran movimiento en la historia de su raza. ${ }^{77}$ 
Thomson consideraba, en efecto, que la fijación hereditaria de un comportamiento individual previamente reforzado en las aves individuales en virtud de premios por ellos percibidos, y su ulterior preservación en el linaje pese a la extinción de dicho refuerzo, era la clave básica para explicar todos los comportamientos migratorios. ${ }^{78}$ Pero, más allá de la aceptación de ese esquema general, lo cierto es que Thomson consideraba que las explicaciones particulares que podían ser construidas para cada caso específico, eran siempre muy especulativas y ninguna le resultaba demasiado convincente. ${ }^{79}$ Que las migraciones tenían causas evolutivas le parecía innegable; pero, conocer esas causas le parecía demasiado difícil. Y eso era así, sobre todo, debido a que, a su entender, la razón de ser de dichos comportamientos, por obvia que fuese, no permitía inferir tales causas.

Más fácil le parecía a Thomson la solución del segundo problema causal: ése que es el tercero de su lista de cuatro y que atañe a las causas que desencadenan el comportamiento migratorio en las aves individuales. Esas causas, conforme decía él, debían buscarse "en el ciclo estacional o en el ciclo fisiológico de la vida del ave en estudio, 0 en ambas cosas"; 80 y eso, claro, las hacía más accesibles a la investigación empírica. Lo importante era no asociar demasiado estrechamente esas causas inmediatas del comportamiento con sus causas evolutivas. Se podría pensar que el estímulo anual debe ser necesariamente de la misma naturaleza de la causa evolutiva; y así, si la escasez de de comida se muestra como no siendo el estímulo anual para la migración, se llegaría a concluir, quizá erróneamente, que ella no ha jugado ningún papel en el origen de ese comportamiento. ${ }^{81}$

Por fin, una vez determinada la causa inmediata que desencadena el comportamiento migratorio, resta aun la solución de un cuarto y último problema que se plantearía al intentar determinar el modus operandi del comportamiento migratorio: "icómo los migrantes encuentran su camino?" ${ }^{82}$ Eso lo deja muy en claro el propio Thomson en la reseña de los cuatro problemas de la migración de aves que él mismo hace en el sumario de su decimosexto capítulo:

\footnotetext{
La migración de las aves es, entonces, la expresión de una costumbre congénita - un ejemplo de comportamiento que supone una capacidad heredada. Es una costumbre costosa y ella no perduraría si no sirviese a alguna meta útil: las ventajas de la costumbre, sin embargo, son en parte obvias. Pero esas ventajas no explican la existencia de la migración: la existencia de ventajas no es, en sí misma, una fuerza causal. La causa última y el origen de la costumbre racial debe ser puesta en la historia pasada de las especies migratorias. Los factores inmediatos, concomitantemente a la existencia de la tendencia heredada, deben también actuar como estimulo recurrente activando la costumbre en el individuo en las ocasiones correspondientes. Conocido el fin útil, la causa originadora y el estimulo desencadenante, el modus operandi de la migración aun queda inexplicado. ${ }^{83}$
}

No debe pensarse, sin embargo, que ese divorcio entre los conceptos de causa remota y presión selectiva que encontramos en Thomson, sea la expresión de un modo pensar generalizado que haya precedido a la ulterior apropiación darwinista de la dicotomía próximo-remoto. Antes de Thomson ya pueden encontrarse ejemplos de ese mismo modo darwiniano de entender la distinción próximo-remoto que después reencontramos en Baker y más tarde en Lack o Mayr. Tal el caso, por ejemplo, del fisiólogo Edward Albert Schäfer ${ }^{84}$ que, en su artículo On the incidence of daylight as a determining factor in bird-migration, publicado por Nature en 1907, también plantea la dicotomía próximo-remoto, homologando causas remotas y presiones selectivas. ${ }^{85}$

\section{Albert Schäfer: el extraño caso del fisiólogo darwinista}

Diferentemente de Thomson, Baker, y Poulton, Schäfer no era lo que hoy caracterizaríamos como un naturalista: ${ }^{86}$ su área de actuación era la Fisiología experimental. ${ }^{87}$ En Gran Bretaña, donde ese dominio disciplinar no se había desarrollado tan rápidamente como lo había hecho en Francia y en Alemania, él fue pionero en esa área de estudios. ${ }^{88}$ En ese sentido, tanto su interés, en los aspectos ecológicos y evolutivos de la migración de las aves, como su compro- 
miso con la Teoría de la Selección Natural, no dejan de ser una rareza. Los fisiólogos de aquellos años, al igual que la mayor parte de los biólogos cuyas áreas de investigación también tenían que ver con la esfera de las causas próximas y se desarrollaban por la vía experimental, solían mostrar cierta dificultad para reconocer el valor de dicha teoría. ${ }^{89}$ Ya vimos a Baker lamentándose por ese asunto; y, aun hoy, bajo la mirada predominantemente laboratorial del fisiólogo experimental, los fenómenos ecológicos y evolutivos siempre tienden a quedar en la penumbra. ${ }^{90}$

Schäfer, en cambio, era decididamente darwinista y su compromiso con la Teoría de la Selección Natural era claro. Eso se hace evidente en el artículo de Nature al que aquí estoy aludiendo. Según ahí leemos, la migración de las aves "sólo es explicable, como cualquier otro fenómeno de la vida [...] por la teoría de la selección natural". ${ }^{11}$ Y puede decirse que, para él, la polaridad próximo-remoto era una exigencia para comprender esa complementariedad entre los enfoques fisiológico y evolucionista de los fenómenos biológicos sobre la que después Mayr tanto insistiría. ${ }^{92}$ Sin ella, Schäfer hubiese tenido dificultades para compatibilizar su oficio de fisiólogo y su reconocimiento del poder explicativo de la Teoría de la Selección Natural. Pero, si el hecho de que el fisiólogo Schäfer se haya interesado por la migración de las aves desde un punto de vista evolutivo no deja de ser algo llamativo, ${ }^{93}$ más sorprendente aun es la fineza de su abordaje. Y creo que esa fineza también está relacionada con la aceptación de la polaridad próximo-remoto.

A Schäfer la explicación de la migración de las aves en virtud de la alternancia estacional en la abundancia y escases de recursos alimenticios no le parecía satisfactoria. ${ }^{94}$ Él prefería una explicación evolutiva que estableciese una relación más inmediata entre esa explicación y aquella que el fisiólogo podía dar al identificar el estímulo desencadenante del comportamiento en estudio. Schäfer prefería una explicación en la cual esa causa próxima estuviese más estrechamente conectada con la presión selectiva, con la causa remota, que había dado origen al comportamiento migratorio, ${ }^{95}$ y la duración del día era un factor que permitía establecer esa conexión: ella podía jugar como estímulo desencadenante del comportamiento ejecutado por los pájaros individuales, pero también podía operar como factor ecológico capaz de dar lugar a una presión selectiva. El acoplamiento entre ambas series causales se hacía así más evidente; y eso podía reforzar la explicación que las combinase.

Las aves predadoras individualizan visualmente a sus presas; y ese es el caso, sobre todo, de las aves migrantes. Para Schäfer, la clave del entrelazamiento entre causas próximas y remotas que explicaba el fenómeno de la migración, estaba ahí. No habiendo desarrollado la visión nocturna que encontramos en aves predadoras no migrantes como las lechuzas, las aves migratorias precisan de días más largos para poder cazar lo necesario para subsistir y para sostener sus crías. ${ }^{96}$ Así, la disminución de las horas de luz que, según parece, estimula el vuelo migratorio en los pájaros individuales, también podría haber generado una dificultad para cazar, capaz, a su vez, de redundar en una presión selectiva favorable a los vuelos migratorios que permitiesen evitar la baja luminosidad de los inviernos. ${ }^{97}$

Sin embargo, aun cuando la lectura de ese paper de Schäfer pone en evidencia que él entendía muy bien la distinción próximo-remoto, en esas páginas no encontramos un tratamiento directo de dicha polaridad: ella es aludida lateralmente y claramente presupuesta, como si fuese algo establecido y reconocido, pero no es explícitamente trazada. En realidad, en lo atinente a esa cuestión Schäfer sólo va un poco más lejos de lo que había ido Darwin en su libro sobre las plantas insectívoras ${ }^{98}$ En esa obra, cuyos asuntos tienen más que ver con la Fisiología que con Biología Evolucionaria o la Auto-Ecología, se usa la expresión `causa próxima ` para aludir a los factores que podrían desencadenar ciertos procesos que ocurren en los tejidos de esas plantas; ${ }^{99}$ y el modo en el que Darwin se refiere a ellos corresponde perfectamente con la caracterización que Mayr nos permite hacer de ese orden causal. Sin embargo, ahí no se plantea ninguna contraposición explícita entre ese tipo de causas y causas de otra índole de carácter específicamente evolutivo. Darwin no llega a ver esa polaridad; aunque sí la pone en evidencia en otra obra de esa misma época.

En sus conclusiones sobre las plantas trepadoras, ${ }^{100}$ Darwin completa sus consideraciones fisiológicas sobre esos vegetales, con hipótesis sobre las presiones selectivas que habrían posibilitado en ellas el ejercicio de la función de trepar y que también habrían determinado los diferentes modos de ejercer esa función que en ellas encontramos. Pero él no recurre a ninguna contraposición clara entre los dos órdenes causales a los que, de hecho, se está refiriendo de modo paralelo y sin confundirlos. Es decir: Darwin se está moviendo dentro de esa polaridad, implícitamente la 
maneja y la respeta, pero no consigue reconocerla: aun cuando le hubiese venido muy bien hacerlo para poder explicar mejor sus propios planteamientos. Darwin, podemos estar seguro de eso, no llegó a entrever con claridad la distinción próximo-remoto; por eso no la encontramos formulada en sus obras. Fueron éstas, sin embargo, las que transformaron a esa polaridad en una exigencia de la razón: fue Darwin que instituyó la Biología de linajes. Antes de él la Biología era una ciencia de organismos: una ciencia de causas próximas.

Sí encontramos la polaridad próximo-remoto, empero, en la introducción que Edward Poulton escribió para sus Essays on evolution: una antología de artículos suyos del período 1889-1907, que fue publicada en 1908. ${ }^{101}$ Sin usar las expresiones 'próximo' y 'remoto', Poulton (1908, p. xlvi) contrapone claramente las preguntas por el cómo (how) de los fenómenos biológicos con las preguntas por su porqué (why); y además de subrayar que ambas formas de interrogación son igualmente legitimas y necesarias, él también subraya que, para entender los hechos biológicos, las dos deben ser planteadas: sin riesgo de interferencia entre las respuestas que vengan a darse para ellas y con la certeza de que tales respuestas deberán iluminarse y sostenerse mutuamente.

Palabras y conceptos no son las mismas cosas. Una palabra como 'adaptación' puede usarse para expresar dos conceptos tan diferentes como lo son el de adaptación fisiológica y el de adaptación evolutiva; ${ }^{102}$ y un mismo concepto puede expresarse con diferentes palabras: en la Biología actual, por ejemplo, 'ontogenia' y 'desarrollo' pueden significar lo mismo. No hay nada de extraño, por eso, en atribuirle a Poulton una comprensión bastante clara de la distinción próximoremoto; aun cuando se esté reconociendo que él no usaba esos términos. Como tampoco creo que se pueda negar que Julian Huxley estuviese reconociendo esa misma distinción cuando, en 1943, él decía que: "todo problema biológico tiene un aspecto evolutivo y un aspecto inmediato, una significación funcional así como una base mecánica, y ambos problemas deben ser estudiados". ${ }^{103}$ Aun cuando él tampoco usase la terminología de las causas próximas y remotas. ${ }^{104}$

\section{En defensa del adaptacionismo}

Lo que pone en evidencia la comprensión cabal que Poulton tenía de la polaridad próximo-remoto - pese a no usar esos términos -, es el contexto en el que la utiliza. Él no recurre a ella, simplemente, para mejor plantear y delimitar un problema y para evitar la superposición obstaculizante entre cuestiones diferentes. Como Mayr, Poulton recurre a ella para defender un tipo de interrogación ${ }^{105}$ y para evitar polémicas desnecesarias: motivadas, solamente, por la no visualización de esa dicotomía entre dos modos de interrogar lo viviente. ${ }^{106}$ Poulton, ${ }^{107}$ además, tiene un interlocutor bien definido, que es un contrincante de peso: John Bretland Farmer (1865-1944), ${ }^{108}$ el gran pionero de la Fisiología Vegetal ${ }^{109}$ y de la Fitopatología ${ }^{110}$ en Gran Bretaña.

En la alocución presidencial que él había proferido ${ }^{111}$ en la sección botánica del encuentro de la British Association for the Advancemen of Science, ocurrido a mediados de 1907, Farmer había criticado al adaptacionismo darwiniano diciendo que:

Se hace un verdadero daño tolerando un acrítico hábito mental, muy común, que consiste en considerar las estructuras como si fuesen respuestas adaptativas a estímulos de diversa índole. No alcanza con explicar la apariencia de una estructura en base a su utilidad; estrictamente hablando esas tentativas lejos de proveer alguna explicación, de hecho tienden a obturar el camino de la indagación justamente donde la investigación científica debería comenzar. ${ }^{112}$

Sin poner en duda "que muchas respuestas a tales estímulos son de una índole tal que hacen al organismo más adaptado a su ambiente", Farmer, como Thomson haría después al distinguir entre la razón de ser de un comportamiento migratorio y su explicación causal, sostenía que "ofrecer el carácter adaptativo de algo como siendo su explicación es anticientífico y superficial". ${ }^{113}$ Y creo que Poulton interpreta correctamente a dónde quería llegar Farmer. Según él, este último quería decir que: 
[...] las explicaciones que la selección natural ofrece del origen y el crecimiento de ciertos rasgos adaptativos de las plantas, no sólo no llegan a explicar los fenómenos, sino que además se interponen en el camino de una investigación sobre las secuencias de hechos por las cuales dichos rasgos se desarrollaron en el individuo. ${ }^{114}$

Y era eso lo que efectivamente estaba en juego: el fisiólogo Farmer sospechaba que la pseudo-explicación histórica darwiniana, centrada en la historia del linaje, ocultase las verdaderas causas del fenómeno organísmico. Interesado en las causas próximas, Farmer desconocía la relevancia de las causas remotas.

Poulton, en cambio, veía con claridad la diferencia existente entre la explicación del mecanismo que opera en un organismo individual y la explicación adaptacionista relativa a la historia evolutiva de ese mecanismo; ${ }^{115}$ y para explicarla recurrió a esta pertinente comparación:

Un revólver moderno, bien construido y cargado, se dispara, para bien o para mal, cuando se le aprieta el gatillo; pero la propia existencia del revólver depende de una larga serie de etapas pasadas, cada una de las cuales fue más ventajosa que su predecesora. El reconocimiento de esa historia no obstaculiza el camino de la indagación, sino que hasta estimula y sugiere un estudio minucioso e inteligente del mecanismo final en toda su complejidad. ${ }^{116}$

Pero, además de tener clara la diferencia entre explicar fenómenos fisiológicos de nivel organísmico y explicar fenómenos evolutivos que ocurren en el plano de los linajes, que como vimos al inicio es un elemento central de la distinción próximo-remoto, Poulton también quería defender lo que puede entenderse como el carácter legítimamente teleológico de las explicaciones por selección natural. ${ }^{117}$ En otro trabajo, Farmer, que pretendía mostrar "cómo la forma orgánica puede ser considerada como el producto necesario de una combinación de materia y fuerza, sin referencia a cualquier explicación teleológica" ${ }^{118}$ había afirmado que "la literatura actual todavía rebosa de explicaciones teleológicas que realmente no explican nada, pero obstaculizan el camino de la investigación científica". ${ }^{19}$ Y Poulton, ${ }^{120}$ que conocía esa diatriba anti-seleccionista, ${ }^{121}$ quería mostrar que "el estudio de la adaptación no cierra el camino de la investigación"; 122 sino que lo ilumina. Su propia experiencia como naturalista de campo y de museo le daba, además, un ejemplo de ello:

Hace unos días estaba analizando algunos ejemplares de mariposas (butterflies) y polillas (moths) que habían sido expuestos a la luz a lo largo de varios años. Noté que, en general, el pigmento de las polillas se había desvanecido mucho más que el de las mariposas. Eso me hizo pensar que los pigmentos estables son más necesarios para las mariposas expuestas a la luz del sol tropical, que para las polillas que vuelan al atardecer o de noche. Por eso, en las mariposas sería seleccionado un grado de estabilidad en los pigmentos mayor al seleccionado en las polillas. El profesor Farmer sostendría, claro, que esa es una de las explicaciones teleológicas que realmente no explican nada y que sobre todo obstaculizan el camino de la indagación científica. Yo, por el contrario, creo que ella explica mucho. Explica la razón (reason) de por qué (why) fueron seleccionados pigmentos con ciertas cualidades, que se transformaron en caracteres de ciertas especies. Ella no explica cómo [how] pudieron surgir pigmentos con las cualidades de esos que fueron seleccionados; pero lejos de cerrárselo, esa sugerencia le señala el camino a la investigación. De hecho, una investigación ulterior sobre la naturaleza química de esos pigmentos, y sobre los pasos a través de los cuales surgen en los individuos, es ahora más probable que antes de que la sugestión fuese hecha. ${ }^{123}$

Eso, dice por otra parte Poulton, ${ }^{124}$ se aplica perfectamente al ejemplo esgrimido por el profesor Farmer en su ya citada alocución de 1907. Este último había dicho que:

En las plantas superiores, una de las respuestas más comunes al estimulo hecho por una herida, es la formación de una capa de corteza sobre el tejido dañado y expuesto. Nadie niega que esa sea una reacción de gran utilidad, dado que ella bloquea la excesiva evaporación de agua y la entrada de organismos parásitos. Y sin embargo yo supongo que nadie iría tan lejos de afirmar seriamente que la obviedad de esa ventaja explica satisfactoriamente por qué se produce la capa de corteza. ${ }^{125}$ 
Pero, conforme Poulton le puede replicar:

Esas ventajas, si su ocurrencia es científicamente comprobada, probablemente explicarían por qué la capacidad de formar corteza fue seleccionada, transformándose en un carácter de las plantas superiores. Ellas [las ventajas] no explican cómo la capa se formó; pero, lejos de cortar el camino, queda muy claro que la prueba de las importantes ventajas conferidas contribuye inmensamente a interesarnos en la corteza, y eso aumenta considerablemente la probabilidad de que el estudioso emprenda una investigación sobre la secuencia de hechos por los que la dicha corteza es generada por la planta individual. ${ }^{126}$

Sin usar las expresiones próximo-remoto, Poulton comprende y muestra la dualidad a la que esa polaridad alude con una claridad que apenas se insinúa en Baker y en los demás predecesores de Mayr que hasta aquí analicé. Lo que Poulton consigue ver es, en primer lugar, la articulación que puede existir entre esas dos sendas de indagación que él también caracteriza en virtud de la oposición entre un cómo y un porqué de las estructuras y de los procesos biológicos:

Los intentos por responder las cuestiones por qué (why) y cómo (how) - a qué fin (to what end) y de qué modo (in what way) - bajo ningún punto de vista interfieren entre sí. Esos dos lados de la investigación, por el contrario, se dan mutua asistencia y estimulo. ${ }^{127}$

Pero, además de eso, él también nos muestra que al accederse al plano de las causas remotas se puede reconocer y entender esa dimensión teleológica de los fenómenos biológicos ${ }^{128}$ que sólo la Teoría de la Selección Natural permite explicar. ${ }^{129}$

Poulton, en este sentido, va claramente más lejos que Mayr: él reconoce esa teleología sin ambages, ${ }^{: 130}$ y al hacerlo pone en evidencia la propia genealogía de la polaridad próximo-remoto. Citando nada menos que a Whewell, ${ }^{131}$ Poulton reivindica las preguntas por qué impugnadas por Farmer, diciendo que "la idea de causa final es una condición esencial cuando se trata de llevar adelante nuestras investigaciones sobre los cuerpos organizados"; ${ }^{132}$ y eso nos remite a lo que podría caracterizarse como la forma primitiva, pre-darwiniana, de la polaridad que nos ocupa. Una forma primitiva cuyas raíces, como dije al inicio, están en los albores de la ciencia moderna.

\section{El ancestro de la polaridad próximo-remoto}

En cierto sentido, y como Mayr ${ }^{133}$ mismo lo sugiere cuando la remonta a Boyle, la distinción entre causas próximas y remotas puede ser considerada como un eco distorsionado de aquella distinción entre causas primeras y causas segundas, o entre causas primeras y causas próximas, o incluso entre causas últimas y causas próximas, que comienza a insinuarse en los albores de la ciencia moderna. ${ }^{134}$ Sin dejar de aparecer en muchos otros autores, ${ }^{135}$ esta polaridad anterior cobra su formulación más clara en la obra de Claude Bernard. ${ }^{136}$ Éste distingue entre causas primeras que explicarían el porqué de los fenómenos naturales, pero no son accesibles a la ciencia natural, y causas próximas que, estando al alcance de la observación y de las manipulaciones experimentales, sólo dan a conocer el cómo de dichos fenómenos. ${ }^{137}$ Pero, mientras en ese contexto las causas últimas estuvieron siempre asociadas a algo que escapaba al dominio de la ciencia natural, la dicotomía evolucionista entre causas próximas y últimas remite, claramente, a dos órdenes de la causación natural. ${ }^{138}$

Pero, obviamente, la semejanza terminológica, que al traducir 'ultimate' por 'remoto' ciertamente se empaña, no es casual, ni es un caso de convergencia. Ella se explica genealógicamente, por filiación compartida: la polaridad evolucionista deriva de la polaridad clásica. Los evolucionistas recurrieron a ella para delimitar y reivindicar sus objetivos cognitivos. Y no lo hicieron porque sí. Lo hicieron porque la Teoría de la Selección Natural le daba eficacia causal a la razón de ser. Bajo su cobertura, la utilidad, la ventaja, podía tener valor explicativo. Lo que el pensamiento teológico le 
adjudicaba a causas últimas de carácter sobrenatural, la teoría formulada por Darwin se lo atribuía a una causa natural y, en ese sentido, segunda. Pero, en la medida en que esa causa se identificaba con la razón de ser de las estructuras y procesos biológicos, ella podía merecer el rótulo de 'causa última'.

Puede decirse, en este sentido, que, históricamente hablando, la polaridad próximo-remoto es un efecto de la naturalización de la teleología operada por el darwinismo ${ }^{139}$. Desde un punto de vista epistemológico, su fundamento es más general: tiene que ver con el propio surgimiento de una Biología de linajes: una Biología cuyo objeto privilegiado ya no es el organismo. Pero, cuando se rastrea cómo fue que esa polaridad comenzó a ser avizorada, se constata que su motivación efectiva fue más limitada: tenía que ver con el hecho de que el darwinismo había dado con un modo no teológico de preguntar ipor qué? De ahí que, en general y salvando la anomalía neolamarckiana representada por Thomson, las causas remotas hayan sido identificadas sólo con las presiones selectivas.

\section{Una última cuestión}

Uno se podría preguntar, por fin, si la historia de la polaridad próximo-remoto pertenece a la Historia de la Ciencia o a la Historia de la Filosofía de la Ciencia, y así, a la Historia de la Filosofía. Quienes la formularon fueron naturalistas preocupados en develar un aspecto del mundo natural y, en este sentido, estudiar esa historia no parece algo demasiado diferente de estudiar la historia del concepto de mimetismo, del concepto de gen, o del concepto de adaptación. Causas remotas y causas próximas, se dirá también, son cosas que están en el mundo natural. Pero la polaridad próximo-remoto tampoco deja de implicar una demarcación en el plano del conocer, una dicotomía en nuestros modos de interrogar; y en este sentido ella parece estar mostrando un aspecto de la gramática de la Biología moderna y no diciendo algo sobre los fenómenos biológicos. Si se atiende a esto último, ella aparece como una distinción epistemológica cuya historia sería objeto de la Historia de la Filosofía.

Creo, sin embargo, que esa dualidad no debe sorprendernos; ni tampoco preocuparnos demasiado. La reflexión epistemológica que llamamos Filosofía de la Ciencia, brota de la propia ciencia: ella no surge de preguntas que se le dirigen a la ciencia desde su exterior. La ciencia es la que llama a esa reflexión para resolver los equívocos que genera su desarrollo y que obstaculizan su progreso. Por eso, la historia de esas elucidaciones forma parte de la Historia de la Ciencia. Sin dejar de ser, sin embargo, parte de la historia de las tesis filosóficas. Aun cuando quienes llegaron a esas tesis sean naturalistas cuyos nombres no consten en el índice de ningún libro de Historia de la Filosofía.

\section{Notas e referências bibliográficas}

Gustavo Caponi es doctor en Lógica \& Filosofía de la Ciencia (UNICAMP, 1993), profesor asociado del Departamento de Filosofía de la Universidade Federal de Santa Catarina, e investigador becario del CNPq. E-mail: gustavoandrescaponi@gmail.com

1 Sobre la carrera de Mayr, y su relevancia, ver: AYALA, Francisco. Ernst Mayr and the theory of evolution. Ludus Vitalis. México: Centro Lombardo Toledano, v. 12, n. 21, p. 3-13, 2004; y DIAMOND, Jared. Ernst Mayr: Obituary. Nature, London: Macmillan, v. 433, n 7027, p. 700-701, 2005.

2 MAYR, Ernst. Cause and effect in Biology. Science, Washington, AAAS, v. 134, p. 1501-1506, 1961. Hay un estudio pormenorizado de este artículo en el segundo anexo de: MAUREL, Marie; MIQUEL, Paul. Programme génétique: concept biologique ou métaphore? Paris: Kimé, 2001. p. 99-110. Mayr insistió en esa distinción en obras posteriores que aquí iré citando.

3 ROGER, Jacques. Biologie du fonctionnement et Biologie de l'évolution. In: BARREAU, Hervé (Ed.). L'explication dans les sciences de la vie. Paris: CNRs, 1983.

4 ARIEW, André. Ernst Mayr's ultimate/proximate distinction reconsidered and reconstructed. Biology \& Philosophy, Dordrecht: Kluwer, v. 18, p. 553-565, 2003. Ejemplos de ese influjo en la Filosofía de la Biología, se pueden encontrar en: JACOB, François. La lógica de lo viviente. Barcelona: Laia, 1970. p. 14; CAPONI, Gustavo. Biología Funcional vs Biología Evolutiva. Episteme, Porto Alegre: ILEA // UFRGS, n. 12, p. 23-46, 2001; WEBER, Marcel. Philosophy of experimental Biology. Cambridge: Cambridge University Press, 2005. p. 3; ROSENBERG, Alexander. Darwinian reductionism. Chicago: Chicago University Press, 2006. p. 16. 

dichotomy still useful? Science. Washington: AAAS, v. 334, p. 1512-1516, 2011.

6 FRANCIS, Richard. Causes, proximate and ultimate. Biology \& Philosophy, v. 5, p. 401-415, 1990.

7 GILBERT, Scott; OPITZ, John; RAFF, Rudolf. Resynthesizing evolutionary and developmental Biology. Developmental Biology, Amsterdam, Elsevier, v. 173, p. 357-372, 1996; AMUNDSON, Ron. The changing role of the embryo in Evolutionary Biology. Cambridge: Cambridge University Press, 2005. p. 225; CALLEBAUT, Werner; MÜLLER, Gerd; NEWMAN, Stuart. The organic systems approach: Evo-Devo and the streamlining of the naturalistic agenda. In: SANSON, Roger; BRANDON, Robert (Ed.). Integrating evolution and development. Cambridge: MIT Press, 2007. p. 26.

8 Ver: LALAND et al, op. cit., p. 1516; FOLGUERA, Guillermo. Enfoques y desenfoques de los programas de investigación en Biología Evolutiva del Desarrollo. Ludus Vitalis. México: Centro Lombardo Toledano, v. 19, n. 35, p. 325-331, 2011; MORANGE, Michel. La vie, l'évolution et l'histoire. Paris: Odile Jacob, 2011a. p. 83.

9 Sea para debilitarla: ARIEW, André. Ernst Mayr's ultimate/proximate distinction reconsidered and reconstructed. Biology \& Philosophy. Dordrecht: Kluwer, v. 18, p. 553-565, 2003. Sea para fortalecerla: CAPONI, Gustavo. La Biología Evolucionaria del Desarrollo como ciencia de causas remotas. Signos Filosóficos. México: UAM, v. 10, n. 20, p. 121-142, 2008.

10 Ver: MORANGE, Michel. What will result from the interaction between functional and evolutionary Biology? Studies in History \& Philosophy of Biological Sciences. Amsterdam: Elsevier, v. 42, p. 69-74, 2011b.

11 Ver: MAYR, Ernst. Una larga controversia: Darwin y el darwinismo. Barcelona: Crítica, 1992. p. 65. También: MAYR, Ernst. Así es la Biología. Madrid: Debate, 1998. p. 134.

12 Sobre Baker, consúltese: WILMER, Ernest; BRUNET, Paul. John Randal Baker. Obituary Notices of Fellows of the Royal Society. London: Royal Society, v. 31 , p. 33-63, 1985.

13 Referencias biográficas sobre Lack pueden encontrarse en: THORPE, William. David Lack. Obituary Notices of Fellows of the Royal Society. London: Royal Society, v. 20, p. 271-293, 1974. Analisé su importancia como pionero de la Ecología Evolucionaria en: CAPONI, Gustavo. La segunda agenda darwiniana. México: Centro Lombardo Toledano, 2011. p. 129-143.

14 Al respecto de Schäfer, ver: HILL, Leonard. Edward Albert Shaffer. Obituary Notices of Fellows of the Royal Society. London: Royal Society, v. 1, n. 4, p. 400-407, 1935.

15 Sobre Thomson, ver: ELLIOTT, Hugh. Obituary of Arthur Landsborough Thomson. Ibis. London: British Ornithologists' Union, v. 12, n. 1, p. 68-72, 1978.

16 BEATTY, John. The proximate/ultimate distinction in the multiple careers of Ernst Mayr. Biology \& Philosophy. Dordrecht: Kluwer, v. 9, p. 333-356, 1994.

17 Cf. CAPONI, Gustavo. Cómo y por qué de lo viviente. Ludus Vitalis. México: Centro Lombardo Toledano, v. 8, n. 14, p. 67-102, 2000.

18 Al respecto de la carrera de Poulton, ver: HALE CARPENTER, Geoffrey. Edward Bagnall Poulton. Obituary Notices of Fellows of the Royal Society. London: Royal Society, v. 5, n 14, p. 655-680, 1945. Sobre la importancia de su obra, ver: BOWLER, Peter. El eclipse del darwinismo. Barcelona: Labor, 1983. p. 208; KIMLER, William. Advantage, adaptativeness, and Evolutionary Ecology. Journal of the History of Biology. Cambridge: Harvard University Press, v. 19, n. 2, p. 215-233, 1986; BOWLER, Peter. Historia Fontana de las ciencias ambientales. México: Fondo de Cultura Económica, 1998. p. 263; CARTON, Yves. Entomologie, Darwin et le Darwinisme. Paris: Hermann, 2011. p. 140; CAPONI, op. cit., 2011, p. 113.

19 Poulton fue uno de los más acérrimos y decididos defensores de la Teoría de la Selección Natural durante ese periodo en torno del 1900 que Julian Huxley llamó de eclipse del darwinismo. Huxley, en efecto, usó esa expresión en: HUXLEY, Julian: La evolución: síntesis moderna. Losada: Buenos Aires, 1965 [1943], p. 22. Bowler la retomó para darle el título a su conocido y aquí ya citado libro sobre ese periodo en el que florecieron teorías que, aceptando la idea de la filiación común y alentando incluso el trabajo empírico a partir de ella, postulaban otros mecanismos del cambio evolutivo diferentes de la selección natural.

20 Sobre esa cuestión historiográfica, ver: CAPONI, op. cit., 2011, p. 114.

21 BAKER, John. The evolution of breeding seasons. In: DE BEER, Gavin (Ed.). Evolution. Oxford: Clarendon Press, 1938.

22 MAYR, Ernst. The growth of biological thought. Cambridge: Harvard University Press, 1982. p. 68. Ver también: MAYR, op. cit., 1992, p. 93; y MAYR, op. cit., 1998, p. 134.

23 FRANCIS, op. cit., 1990, p. 406; BEATTY, op. cit., 1994, p. 346.

24 Sobre la oposición organismo-linaje, ver: CAPONI, Gustavo. Linajes y sistemas: dos tipos de individuos biológicos. Scientiae Studia. São Paulo: USP, v. 10, n. 2, p. 243-268, 2012.

25 CAPONI, Gustavo. El concepto de presión selectiva y la dicotomía próximo-remoto. Aurora. Curitiba: PUC Paraná, v. 25, n. 36, p. 197-216, 2013.

26 Cf. MAYR, op. cit., 1982, p. 68; y op. cit., 1998, p. 133.

27 "Ecología de los organismos o especies individuales; idioecología; autoecología" (LINCOLN, Roger; BOXSHALL, Geoffrey; CLARK, Paul. Diccionario de Ecología, evolución y Taxonomía. México: Fondo de Cultura Económica, 2009, p. 66. La Autecología podría ser practicada por un naturalista creacionista, ajeno a cualquier perspectiva evolucionista. Lo mismo vale para una Ecología de Poblaciones que no considere factores y fenómenos evolutivos. El estudio del impacto de un incremento en la densidad de una población de depredadores en la densidad de la población de sus presas, puede ser hecho sin aludir a procesos evolutivos y a causas remotas.

28 Sobre el carácter proximal de la Autecología y de la simple Ecología de Poblaciones, ver: ORIANS, Gordon. Natural selection and ecological theory. The American Naturalist. Chicago: The University of Chicago Press, n. 890, p. 257-263, 1962. También: COLLINS, James. Evolutionary Ecology and the use of natural selection in Ecological Theory. In: KELLER, David; GOLLEY, Frank (Ed.). The Philosophy of Ecology. London: The University of Georgia Press, 2000, p. 296.

29 Cf. CAPONI, op. cit., 2008, p. 129-132.

30 Sobre la diferencia entre ambas cosas, ver: CAPONI, Gustavo. Função e desenho na Biologia contemporânea. São Paulo: Editora 34 // Scientiae Studia, 2012. p. 58-61. Cuando Richard Francis afirma que la distinción próximo-remoto es importante para la Ecología, eso sólo puede valer para la Ecología Evolucionaria. (Cf. FRANCIS, op. cit., 1990, p. 401) 

en las poblaciones entendidas como linajes, también permite reconocer el carácter de causa remota a los constreñimientos ontogenéticos de la evolución que hoy estudia la Biología Evolucionaria del Desarrollo. Al respecto, ver lo que afirmé en la nota 19 de este trabajo, y también en: CAPONI, op. cit., 2008, p.136-137.

32 Cf. CAPONI, op. cit., 2008, p. 132-35.

33 MAYR, op. cit., 1961, p. 1502. Ver también: BEATTY, op. cit., 1994, p. 334.

34 MAYR, op. cit., 1961, p. 1502; MAYR, op. cit., 1982, p. 68; y MAYR, op. cit., 1998, p. 135.

35 MAYR, op. cit., 1982, p. 69.

36 MAYR, op. cit., 1998, p. 137.

37 MAYR, Ernst. Change of genetic environment and evolution. In: HUXLEY, Julian; HARDY, Alister; FORD, Edmund (Ed.). Evolution as a process. London: Allen \& Unwin, 1954. p. 158.

38 CAPONI, op. cit., 2008, p. 125

39 Cf. CAPONI, op. cit., 2012, p. 254.

40 FOX KELLER, Evelyn. Beyond the gene but beneath the skin. In: OYAMA, Susan; GRIFFITHS, Paul; GRAY, Russell (Eds.). Cycles of contingency. Cambridge: MIT Press, 2001, p. 302. Maurel y Miquel llegaron a decir que, pese haber tenido una cierta fertilidad heurística en los orígenes de la Genética Molecular, la idea de programa genético se había transformado en un obstáculo epistemológico (MAUREL; MIQUEL, op. cit., 2001, p. 89).

41 FOX KELLER, op. cit., 2001, p. 303; BARBEROUSE, Anouk; MORANGE, Michel; PRADEU, Thomas. Introduction to BARBEROUSE, Anouk; MORANGE, Michel; PRADEU, Thomas (Ed.). Mapping the future of Biology. Berlin: Springer, 2009, p. 3. De hecho, según Maurel y Miquel, fue el propio Mayr quien, en 1961 y simultáneamente con François Jacob y Jacques Monod, acuñó esa metáfora (MAUREL; MIQUEL, op. cit., 2001, p. 39).

42 GAYON, Jean. Darwin et l'après Darwin. Paris: Kimé, 1992, p. 335; CAPONI, op. cit., 2011, p. 130.

43 AMUNDSON, op. cit., 2005, p. 201; BEATTY, op. cit., 1994, p. 347.

44 Ver: MAYR, Ernst. Evolution and the diversity of life. Cambridge: Harvard University Press, 1976. p. 312; y MAYR, Ernst. Toward a new Philosophy of Biology. Cambridge: Harvard University Press, 1988. p. 140.

45 MAYR, op. cit., 1982, p. 68. Más aún: Mayr llegó a preferir 'evolutionary cause' por sobre 'ultimate cause'; que es la expresión que estoy traduciendo por 'causa remota'. Al respecto ver: MAYR, Ernst. Proximate and ultimate causations. Biology \& Philosophy, Dordrecht, Kluwer, v. 8, p. 93-94, 1993.

46 MAYR, Ernst. What evolution is. New York: Basic Books, 2001. p. 98-99.

47 MAYR, op. cit., 1961, p. 1502; op. cit., 1982, p. 69; y op. cit., 1998, p. 86.

48 A este respecto, Mayr afirma que: "la pregunta ¿por qué?, en el sentido de ipara qué?, no tiene sentido en el mundo de los objetos inanimados. Uno puede preguntarse ¿Por qué el sol calienta? pero sólo en el sentido de ¿cómo ocurre? Por el contrario, en el mundo viviente la pregunta ¿para qué? tiene un valor heurístico poderoso" (MAYR, op. cit., 1982, p. 72). Y es en el por qué como para qué, que Mayr piensa cuando afirma que fue con Darwin que las preguntas ipor qué? entraron en la ciencia natural (MAYR, op. cit., 1998, p. 134). Como muchos otros evolucionistas, Mayr siempre se va a debatir entre un reconocimiento subrepticio de la teleología naturalizada propia del darwinismo y, por otro lado, su negación explícita. Fundada esta última negación, sobre todo, en una idea limitada de lo que ha de entenderse por 'explicación teleológica'. Al respecto, ver: MAYR, op. cit., 1961, p. 1503; y op. cit., 1988, p. 59. Sobre el modo darwinista de preguntar ¿para qué?, ver: BRANDON, Robert. Concepts and methods in Evolutionary Biology. Cambridge: Cambridge University press, 1996. p. 33.

49 CAPONI, op. cit., 2011, p.136-137.

50 Cf. LACK, David. The natural regulation of animals numbers. Oxford: Clarendon Press, 1954. p. 47; 59; 236; 273. Al respecto, ver: MAYR, op. cit., 1982 , p. 68); y BEATTY, op. cit., 1994, p. 346.

51 Por lo que apunta Beatty, Mayr y Lack pueden haber madurado conjuntamente esta recuperación de la distinción próximo-remoto (BEATTY, op. cit., 1994, p. 346.). Pero, de hecho, Mayr termina por concederle la prioridad a Lack (MAYR, op. cit., 1993, p. 94).

52 LACK, op. cit., 1954, p. 5.

53 LACK, op. cit., 1954, p. 5.

54 LACK, David. Evolutionary Ecology. Journal of Ecology, London, British Ecological Society, v. 53, n. 2, p. 237-245, 1965.

55 Lack usa la expresión 'why-and-wherefore' (LACK, op. cit., 1954, p. 62).

56 Ver: BEATTY, op. cit., 1994, p. 348-350. Ése ha sido, por otra parte, uno de los usos más claros de la distinción próximo-remoto. Véase, por ejemplo: JACOB, op. cit., 1970, p. 14; y MORANGE, op. cit., 2011a, p. 10.

57 CAPONI, op. cit., 2011, p. 138.

58 Al respecto, ver: COLLIN, James; BEATTY, John; MAIENSCHEIN, John. Between Ecology and Evolutionary Ecology. Journal of the History of Biology, v. 19, $n$ 2, p.169-180, 1986. También: COLLINS, op. cit., 2000, p. 295. El texto que Gordon Orians publicó en el American Naturalist, en 1962, documenta y explica ese impacto temprano del paper de Mayr (ORIANS, op. cit., 1962, p. 260).

59 BEATTY, op. cit., 1994, p. 345.

60 BAKER, op. cit., 1938, p. 161.

61 BEATTY, op. cit., 1994, p. 345-346.

62 BAKER, op. cit., 1938, p. 162.

63 THOMSON, Arthur. Problems of bird-migration. Boston: Mifflin, 1926.

64 BEATTY, op. cit., p. 342-344.

65 Cf. THOMSON, op. cit., 1926, p. 285. 
THOMSON, op. cit., 1926, p. 259-272.

67 THOMSON, op. cit., 1926, p. 264.

68 THOMSON, op. cit., 1926, p. 261. Thomson usa la palabra 'racial' para referirse a la estirpe, al linaje, en general. Estamos hablando de una época en la que no hay una terminología estandarizada para expresar esos conceptos

68 THOMSON, op. cit., 1926, p. 262.

70 THOMSON, op. cit., 1926, p. 262.

71 THOMSON, op. cit., 1926, p. 264.

72 THOMSON, op. cit., 1926, p. 271.

73 THOMSON, op. cit., 1926, p. 273.

74 THOMSON, op. cit., 1926, p. 262.

75 THOMSON, op. cit., 1926, p. 262.

76 THOMSON, op. cit., 1926, p. 262.

77 THOMSON, op. cit., 1926, p. 273-274.

78 THOMSON, op. cit., 1926, p. 284.

79 THOMSON, op. cit., 1926, p. 262.

80 THOMSON, op. cit., 1926, p. 263.

81 THOMSON, op. cit., 1926, p. 263.

82 THOMSON, op. cit., 1926, p. 263.

83 THOMSON, op. cit., 1926, p. 264.

84 Cf. BEATTY, op. cit., 1994, p. 342.

85 SCHÄFER, Edward. On the incidence of daylight as a determining factor in bird-migration. Nature, London, Macmillan, v. 77, n. 1990, p. 159-163, 1907.

86 Sobre la idea actual de naturalista, que aúna de un modo no del todo preciso al ecólogo y al biólogo evolucionario cuando sus investigaciones exigen el trabajo de campo, ver: BATES, Marston. The nature of natural history. Princeton: Princeton University Press, 1990. p. 7; GRANT, Peter. What does it mean to be a naturalist at the end of the twentieth century? The American Naturalist, Chicago, The University of Chicago Press, v. 155, n. 1, p. 1-12, 2000. Analisé la cuestión en: CAPONI, Gustavo. Georges Cuvier: un fisiólogo de museo. México: UNAM, 2008. p. 131 y ss; y también en: CAPONI, op. cit., 2011, p. 144 y ss.

87 HILL, op. cit., 1935, p. 403.

88 HILL, op. cit., 1935, p. 401.

89 Véase: MAYR, op. cit., 1982, p. 73; y también: MAYR, Ernst. Some thoughts on the History of the evolutionary synthesis. In: MAYR, Ernst; PROVINE, William (Ed.). The evolutionary synthesis: A perspective on the unification of Biology. Cambridge: Harvard University Press, 1980. p. 9-10.

90 Al respecto, ver: BEATTY, John. Evolutionary anti-reductionism: Historical reflections. Biology \& Philosophy, Dordrecht, Kluwer, v. 5, p. 199-210, 1990; y LEWONTIN, R. The bases of conflict in biological explanation. Journal of the History of Biology, Cambridge, Harvard University Press, v. 2, n. 1, p. 35-45, 1969.

91 SCHÄFER, op. cit., 1907, p. 159.

92 Cf. MAYR, op. cit., 1982, p. 73; op. cit., 1998, p. 136.

93 No hay caso: la explicación la migración de las aves parece ser el locus privilegiado para el planteamiento de la polaridad próximo-remoto: así estamos viendo que ocurre en Schäfer, pero también vimos que ese era el caso de Thomson, de Baker y de Lack. Y ese es el ejemplo al que Mayr apela cuando se refiere a aquella curruca de New Hampshire (Cf. MAYR, op. cit., 1961, p. 1502-1503). Pero tanto Mayr cuanto Lack cometen el error de atribuir la distinción a Baker; ignorando a Thomson (Cf. LACK, op. cit., 1965, p. 238; MAYR, op. cit., 1982, p. 68). Es de notar que, en The science of Ecology, Richard Brewer también introduce la distinción entre ambos órdenes causales, usando como ejemplo a la migración de aves y también remitiendo la distinción a Baker (Cf. BREWER, Richard. The science of Ecology. Philadelphia: Saunders \& Harcourt, 1994. p. 29-30). Inevitablemente, la pregunta por la causa de cualquier comportamiento nos pone al borde de una confusión, por lo menos verbal, entre el plano de las causas remotas y el plano de las causas próximas. Por eso ella también llama al esclarecimiento del tópico y puede servirle como ejemplo privilegiado (Véase, por ejemplo: ALCOCK, John. Comportamento animal: uma abordagem evolutiva. Porto Alegre: Artmed, 2011. p. 29). Queda claro, sin embargo, que ella se aplica a toda estructura biológica.

94 SCHÄFER, op. cit., 1907, p. 160-161.

95 SCHÄFER, op. cit., 1907, p. 160.

96 SCHÄFER, op. cit., 1907, p. 161.

97 SCHÄFER, op. cit., 1907, p. 162.

98 DARWIN, Charles. Plantas insectívoras. México/Madrid: UNAM/CSIC, 2008 [1875]

99 DARWIN, op. cit., 2008 [1875], p. 72.

100 DARWIN, Charles. Los movimientos y hábitos de las plantas trepadoras. México/Madrid: UNAM/CSIC, 2009 [1875]. p. 181 y ss.

101 POULTON, Edward. Mutation, Mendelism and Natural Selection. In: . Essays on evolution: 1889-1907. Oxford: Clarendon Press, 1908.

Cf. GRIFFITHS, PAUL. Adaptation and adaptationism. In: WILSON, Robert; KEIL, Frank (Ed.). The MIT Encyclopedia of the Cognitive Sciences. Cambridge: MIT Press, 1999. p. 3

103

HUXLEY, op. cit., 1965 [1943], p. 393. 
Es digno de observarse que Poulton, Huxley y Baker, tuvieron vinculaciones profesionales bastante estrechas. Se puede decir, además, que ambos eran parte de un mismo colegio invisible: un clan que sostuvo la Teoría de la Selección Natural cuando ella era bastante cuestionada y que contribuyó a la articulación del movimiento que desembocó en la consolidación de la Nueva Síntesis. Baker fue discípulo de Julian Huxley (Cf. WILMER; BRUNET, op. cit., 36); y en el libro en homenaje a Edwin Goodrich, organizado por Gavin de Beer, en el que fue publicado el célebre, y aquí discutido, trabajo de Baker sobre la evolución de las temporadas de cría, hay un escrito de Huxley y otro de Poulton (HUXLEY, Julian. The present standing of the theory of sexual selection. In: DE BEER, Gavin (Ed.), Evolution. Oxford: Clarendon Press, 1938 y POULTON, Edward. Insect adaptation as evidence of evolution by natural selection. In: DE BEER, Gavin (Ed.), Evolution. Oxford: Clarendon Press, 1938.

Para Mayr, en efecto, muchas controversias biológicas son sólo un resultado de la confusión entre las dos biologías (Cf. MAYR, op. cit., 1982, p. 73; MAYR, op. cit., 1998, p. 136)

POULTON, op. cit., 1908, p. xliv.

Sobre la carrera de Farmer, ver: BLACKMAN, Vernon. John Bretland Farmer. Obituary Notices of Fellows of the Royal Society, London, Royal Society, v. 5 , n. 14, p. 17-31, 1945.

BLACKMAN, op. cit., 1945, p. 17.

BLACKMAN, op. cit., 1945, p. 20-21.

FARMER, John. Presidential address at the Botany section. In: Seventy-seventh Meeting Of The British Association For The Advancement Of Science, 1907, Leicester 31 July - 7 Aug. Report... London: Murray, 1908, p. 674-683.

FARMER, op. cit., 1908, p. 675.

FARMER, op. cit., 1908, p. 675. Ver: POULTON, op. cit., 1908, p. xliv n.1.

POULTON, op. cit., 1908, p. xliv.

POULTON, Edward, op. cit., 1908, p. 48-94, p. 74 n. 2.

POULTON, op. cit., 1908, p. 74 n. 2. En realidad, esta nota de Poulton es un eco de aquellas líneas de Sobre el origen de las especies en las que Darwin dice que la teoría por él enunciada nos lleva a considerar que "toda estructura compleja y cada instinto es el resultado de múltiples estratagemas [contrivances], cada una de ellas útil para su portador"; es decir: "aproximadamente de la misma manera en la que miramos a cualquier invención mecánica considerándola como el resultado del trabajo, la experiencia, la razón e inclusive de los yerros de muchos trabajadores" (Ver: DARWIN, Charles. On the origin of species. London: Murray, 1859. p.485-486).

117 Poulton retomó esa cuestión en: POULTON, Edward. The value of colors in the struggle for life. In: SEWARD, Albert (Ed.). Darwin and modern sicience. Cambridge: Cambridge University Press, 1910. p. 272-274.

118 FARMER, John. On stimulus and mechanisms as factors in organization - Part II. The New Phytologist, London, The New Phytologist Trust, v. 2, n. 10, p. 217-225, 1903a. La primera parte de este trabajo, que reproduce una conferencia dictada en Southport, durante la reunión de la British Association for the Advancement of Science de 1903, había sido publicada en el número anterior de esa misma Revista: FARMER, John. On stimulus and mechanisms as factors in organization - Part I. The New Phytologist, London, The New Phytologist Trust, v. 2, n. 9, p. 193-201, 1903b.

FARMER, op. cit., 1903a, p. 225.

POULTON, op. cit., 1908 p. xlv; POULTON, op. cit. 1908, p. 74 n2.

Farmer es un exponente característico de la onda anti-seleccionista que Bowler retrata en El eclipse del Darwinismo. Sin negar la teoría de la filiación común, Farmer prefería las tesis mutacionistas de De Vries (Cf. FARMER, op. cit., 1903a, p. 224). Él, conjuntamente con Arthur Darbishire, fue uno de los traductores de Die mutationstheorie (BLACKMAN, op. cit., p. 24); cuya edición en inglés apareció en dos volúmenes publicados entre 1909 y 1910: DE VRIES, Hugo. The mutation theory. Chicago: Open Court, 1919. v. I.; y DE VRIES, Hugo. The mutation theory. Chicago: Open Court, 1910. v. II. Sobre la influencia de De Vries en el pensamiento evolucionista de inicios del siglo XX, ver: BOWLER, El eclipse del darwinismo, op. cit., p. 216 y ss. Sobre la trayectoria de Arthur Darbishire, ver: MARTINS, Lilian. Darbishire, Bateson e Weldon: a controversia sobre a hereditariedade em camundongos (19021904). Filosofia e Historia da Biologia. São Paulo: ABFHIB, v. 3, p. 213-240, 2008, p. 215.

POULTON, op. cit., 1908, p. xliv.

POULTON, op. cit., 1908, pp. xliv-xlv. Poulton está aludiendo ahí a lo que fue el asunto privilegiado de sus investigaciones como naturalista de campo: el estudio sobre el valor adaptativo, y sobre todo mimético, de las coloraciones animales. Las mismas quedaron reflejadas en innúmeros trabajos; y sobre todo en su libro: The colours of animals. New York: Appleton \& Co, 1890. Al respecto, ver: BOWLER, op. cit., 1998, p. 260; KIMLER, op. cit., 1986, p. 217; y CAPONI, op. cit., 2011, p. 113. Sobre la centralidad de la temática del mimetismo en los albores del programa adaptacionista, ver: BOWLER, op. cit., 1983, p. 39; y CAPONI, op. cit., 2011, p. 88

POULTON, op. cit., 1908, p. xlv.

FARMER, op. cit., 1908, p. 676.

POULTON, op. cit., 1908, p. xvi.

POULTON, op. cit., 1908, p. xvi.

GRIFFITHS, op. cit., 1999, p. 3

Al respecto, ver: CAPONI, Gustavo. Teleología naturalizada: Ios conceptos de función, aptitud y adaptación en la Teoría de la Selección Natural. THEORIA. San Sebastián: Universidad del País Vasco, n. 76, p. 95-114, 2013.

Al respecto ver la nota 48

WHEWELL, William. The philosophy of the inductive sciences, v. I. London: Parker, 1847. p. 620.

POULTON, op. cit., 1908, p. xlvi n. 1.

MAYR, op. cit., 1992, p. 65. 
134 SOLÍS, Carlos. Introducción y notas. In: BOYLE, Robert. Física, Química y Filosofía Mecánica. Madrid: Alianza, 1985. p. 196 n. 6.

135 BURTT, Edwin. Los fundamentos metafísicos de la ciencia moderna. Buenos Aires: Sudamericana, 1960. p. 106-113.

136 BERNARD, Claude. Introduction a l'étude de la médecine expériméntale. Paris: Flammarion, 1985 [1865]. p. 124-126.

137 Cf. GRMEK, Mirko. Les legs de Claude Bernard. Paris: Fayard, 1997. p. 42; CAPONI, Gustavo. Claude Bernard y los límites de la Fisiología Experimental. História, Ciências, Saúde - Manguinhos. Rio de Janeiro: Casa de Oswaldo Cruz, v. 8, n. 2, p. 375-406, 2001.

138 MAYR, op. cit., 1993, p. 94.

139 Cf. CAPONI, Gustavo. ¿Fue o no fue Darwin el Newton de la brizna de hierba? Principia. Florianópolis: NEL // UFSC, v. 16, n. 1, p. 53-79, 2012.

[Recebido em Fevereiro de 2013. Aceito para publicação em Abril de 2014] 